\title{
Magnetic detection electrical impedance tomography with total variation regularization
}

\author{
Liling $\mathrm{Hao}^{\mathrm{a}}$, Gang $\mathrm{Li}^{\mathrm{b}}$ and Lisheng $\mathrm{Xu}^{\mathrm{a}}{ }^{\text {,* }}$ \\ ${ }^{a}$ Department of Sino-Dutch Biomedical and Information Engineering, Northeastern University, \\ Shenyang 110819, China \\ ${ }^{b}$ College of Precision Instrument and Opto-electronics Engineering, Tianjin University, Tianjin 30072, \\ China
}

\begin{abstract}
Magnetic detection electrical impedance tomography (MDEIT) is an imaging modality that aims to reconstruct the cross-sectional conductivity distribution of a volume from the magnetic flux density surrounding an object. The MDEIT inverse problem is inherently ill-posed, necessitating the use of regularization. The most commonly used $L^{2}$ norm regularizations generate the minimum energy solution, which blurs the sharp variations of the reconstructed image. Consequently, this paper presents the total variation (TV) regularization to preserve discontinuities and piecewise constancy of the MDEIT reconstructed image. The primal dual-interior point method (PD-IPM) is employed for minimizing the TV penalty in this paper. The proposed method is validated by MDEIT simulated data. In comparison with the performance of $L^{2}$ norm regularization, the results show that TV regularized algorithm produces sharper images and has better robustness to noise. The TV regularized algorithm preserves local smoothness and piecewise constancy, leading to improvements in the localization of the reconstructed conductivity images in MDEIT.
\end{abstract}

Keywords: Magnetic detection electrical impedance tomography, inverse problem, regularization, total variation, primal dual-interior point method

\section{Introduction}

Magnetic detection electrical impedance tomography (MDEIT) is an experimental imaging technique that aims to compute the cross-sectional conductivity distribution of a volume from the magnetic flux density detected around the object. The conductivities of biological tissues contain the anatomic, physiological and pathological information [1]. The conductivity can provide the diagnostically valuable information of the human body compared with CT and MRI. However, electrical impedance tomography (EIT) detects the surface voltage by electrodes on the surface, leading to more errors and less measurements. Since measurements of the magnetic flux density are contactless, MDEIT can record a greater number of measurements with precise detector positions.

\footnotetext{
${ }^{*}$ Corresponding author: Lisheng Xu, Sino-Dutch Biomedical and Information Engineering School, Northeastern University, No. 11, Lane 3, Wenhua Road, Heping District, Shenyang City, Liaoning Province, China. Tel.: 86-24-83683200; Fax: 8624-83681955; E-mail: xuls@bmie.neu.edu.cn.
} 
The inverse problem of MDEIT is ill-posed. Consequently, the regularization algorithms have been applied to the image reconstruction $[2,3]$. However, these $L^{2}$ norm regularization methods limit the capability of describing sharp variations in conductivity and tend to smooth out edges in images. The human images have clear organ boundaries that present sharp variations in conductivity. The ability of reconstructing sharp changes should lead to a better estimation of the boundaries and a better accuracy of the estimated values. The $L^{2}$ norm regularizations blur the boundaries and make it difficult to estimate the size and the contrast value. The paper investigates a regularization method that preserves the edge information in the reconstructed conductivity image.

The total variation (TV) minimizing function regularization preserves discontinuities in the reconstructed profiles [4]. Therefore, the TV regularization is employed to reconstruct the images in MDEIT. The TV is defined as the $L^{1}$ norm of the differences between neighboring pixels. This particular form of the TV penalty enforces sparsity on pixel differences and consequently tends to generate images with piecewise constant regions and sharp boundaries.

\section{Magnetic detection electrical impedance tomography}

The MDEIT system consists of two aspects: forward problem and inverse problem. The forward problem computes the magnetic flux density $\mathbf{B}$ surrounding the object from the known conductivity distribution $\sigma$ according to the boundary value problem and Biot-Savart law $[5,6]$

$$
\left\{\begin{array}{l}
\nabla \cdot \sigma \nabla \varphi=0 \quad \text { in } \Omega \\
-\sigma \frac{\partial \varphi}{\mathbf{n}}=\mathbf{n} \cdot \mathbf{J}_{I} \quad \text { on } \partial \Omega \\
\mathbf{J}=-\sigma \nabla \varphi \\
\mathbf{B}\left(\mathbf{r}^{\prime}\right)=\frac{\mu_{0}}{4 \pi} \int_{\Omega} \mathbf{J}(\mathbf{r}) \times \frac{\mathbf{r}^{\prime}-\mathbf{r}}{\left|\mathbf{r}^{\prime}-\mathbf{r}\right|^{3}} d \nu
\end{array}\right.
$$

For the inverse problem of MDEIT, Ireland just constructed the current density image. The conductivity distribution reconstruction is remained to be solved [3]. In this paper, the reconstruction of the conductivity image is described. The inverse problem of MDEIT can be viewed as seeking a solution of the optimization problem.

$$
\hat{\boldsymbol{\sigma}}=\arg \min _{\boldsymbol{\sigma}} \frac{1}{2}\|F(\boldsymbol{\sigma})-\mathbf{B}\|_{2}^{2}
$$

where $F(\boldsymbol{\sigma})$ is the forward operator. Regularization is a method that uses well-conditioned approximation including proper side constraints to generate stabilized solution. The well-known method is Tikhonov regularization, in which the residual norm and the $L^{2}$ norm penalty are combined with the cost function [7] 


$$
\hat{\boldsymbol{\sigma}}=\arg \min _{\boldsymbol{\sigma}} \frac{1}{2}\|F(\boldsymbol{\sigma})-\mathbf{B}\|_{2}^{2}+\frac{\lambda}{2}\|\mathbf{L} \boldsymbol{\sigma}\|_{2}^{2}
$$

where $\lambda$ is a positive regularization parameter, $\mathbf{L}$ is a regularization matrix. The optimization problem is solved iteratively based on the Gauss-Newton method, obtaining the updated equation as follows:

$$
\Delta \boldsymbol{\sigma}_{k}=-\left(\mathbf{A}_{k}^{T} \mathbf{A}_{k}+\lambda \mathbf{L}^{T} \mathbf{L}\right)^{-1} \cdot\left[\mathbf{A}_{k}^{T}\left(\mathbf{F}\left(\boldsymbol{\sigma}_{k}\right)-\mathbf{B}\right)+\lambda \mathbf{L}^{T} \mathbf{L} \boldsymbol{\sigma}_{k}\right]
$$

where $k$ is the iteration number, $\Delta \boldsymbol{\sigma}_{\mathrm{k}}$ is the conductivity update, and $\mathbf{A}_{\mathrm{k}}$ is the Jacobian matrix.

The conductivity of biological tissues changes violently for different tissues, physiological and pathological states, corresponding to the sharp edges among different tissues. Therefore, the reconstructed conductivity image should have the discontinuous profile. However, $L^{2}$ norm regularization stabilizes the inverse problem by blurring the sharp edges among blocky structures. The TV regularization can generate images with piecewise constant region and sharp boundary.

\section{Total variation regularization}

The TV regularization was first proposed for the image denoising [8]. This regularization method improves the ill-conditioned nature of the corresponding coefficient matrix, and recovers discontinuous images. For the conductivity image $\boldsymbol{\sigma}$, the TV is defined as

$$
\operatorname{TV}(\boldsymbol{\sigma})=\int_{\Omega}|\nabla \boldsymbol{\sigma}| d \Omega=\sum_{i}\left|\sigma_{m(i)}-\sigma_{n(i)}\right|=\sum_{i}\left|\mathbf{L}_{i} \boldsymbol{\sigma}\right|
$$

where $\nabla \boldsymbol{\sigma}$ is the step change of conductivity between the neighboring element pair, $m(i)$ and $n(i)$ are element indices of the $i$ th neighboring element pair. The TV penalty cannot be straightforward minimized because of its non-differentiability. To remove its non-differentiability, TV side constraint can be approximated as smoothed TV penalty

$$
\mathrm{TV}(\boldsymbol{\sigma}) \approx \mathrm{TV}_{\delta}(\boldsymbol{\sigma})=\sum_{i} \sqrt{\left|\mathbf{L}_{i} \boldsymbol{\sigma}\right|^{2}+\delta}
$$

where $\delta$ is a constant with a small positive value, which enforces the differentiability of the TV penalty. Then the cost function of the inverse problem regularized by the approximate TV penalty is

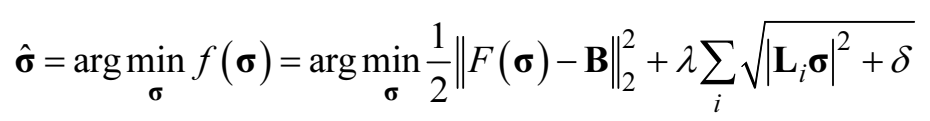


Several different algorithms have been proposed for EIT with TV regularization, for example, steepest descent, Markov Chain Monte Carlo (MCMC), and Primal Dual-Interior Point Method (PDIPM) [9-11]. The PD-IPM does not require the control of the parameter $\delta$, and results in a better approximation of the TV penalty [11]. PD-IPM is applied to MDEIT with TV regularization and the updates of the primal and dual variables can be obtained.

$$
\left\{\begin{array}{l}
\Delta \boldsymbol{\sigma}_{k}=-\left(\mathbf{A}_{k}^{T} \mathbf{A}_{k}+\lambda \mathbf{L}^{T} \mathbf{P}_{k}^{-1} \mathbf{Q}_{k} \mathbf{L}\right)^{-1} \cdot\left[\mathbf{A}_{k}^{T}\left(F\left(\boldsymbol{\sigma}_{k}\right)-\mathbf{B}\right)+\lambda \mathbf{L}^{T} \mathbf{P}_{k}^{-1} \mathbf{L} \boldsymbol{\sigma}_{k}\right] \\
\Delta \mathbf{x}_{k}=-\mathbf{x}_{k}+\mathbf{P}_{k}^{-1} \mathbf{L} \boldsymbol{\sigma}_{k}+\mathbf{P}_{k}^{-1} \mathbf{Q}_{k} \mathbf{L} \Delta \boldsymbol{\sigma}_{k}
\end{array}\right.
$$

With

$$
\eta_{i}^{k}=\sqrt{\left|\mathbf{L}_{i} \boldsymbol{\sigma}_{k}\right|^{2}+\delta} \quad \mathbf{P}_{k}=\operatorname{diag}\left(\eta_{i}^{k}\right) \quad \mathbf{Q}_{k}=\operatorname{diag}\left(1-\frac{x_{i}^{k} \mathbf{L}_{i} \boldsymbol{\sigma}_{k}}{\eta_{i}^{k}}\right)
$$

\section{Simulations and results}

In this section, the performance of the TV regularization algorithm was evaluated and compared with the $L^{2}$ norm penalty. The evaluation was conducted on 2D and 3D simulated data. The 2D simulated phantom is shown in Figure 1(a). The background conductivity was set at $1 \mathrm{~S} / \mathrm{m}$, and the small inclusions presented the same value of $2 \mathrm{~S} / \mathrm{m}$. The $10 \mathrm{~mA}$ current was injected by one pair of opposite electrodes shown in Figure 1(b). The total element number is 2160 for simulating the magnetic flux density data (forward model) and 540 for the image reconstruction (inverse model). Figure 1(c) shows the positions of the magnetic flux density measurement coils at 120 equally spaced angles along three equally spaced circles surrounding the circular imaging object, which results in 360 measurements. For 2D simulated phantom, only magnetic flux density $B_{z}$ can be recorded by coils at these positions, so the orientations of coils are displayed as Figure 1(c).

In order to validate the feasibility of the TV regularization for 3D MDEIT reconstruction, a simple $3 \mathrm{D}$ cubic model with the size $20 \times 20 \times 3 \mathrm{~cm}^{3}$ was constructed to perform the simulation, which is shown in Figure 2(a). The $10 \mathrm{~mA}$ current was injected by the electrodes shown in Figure 2(a). The forward problem was calculated on the finer mesh with 7200 elements, while the conductivity images were reconstructed on the coarse mesh with 1200 elements of size $1 \mathrm{~cm} \times 1 \mathrm{~cm} \times 1 \mathrm{~cm}$. The background conductivity of the model was $1 \mathrm{mS} / \mathrm{cm}$. The simulated phantom had two inclusions with different conductivities $2 \mathrm{mS} / \mathrm{cm}$ and $0.5 \mathrm{mS} / \mathrm{cm}$ in the top and bottom parts, respectively. Figure 2(b) shows the tomographic conductivity map of the phantom. The "measured" magnetic flux density data $B_{y}$ were obtained by calculating the forward problem. The positions of the magnetic flux density measurement points are displayed in Figure 2(c). All the measurement points located in the six planes surrounding the imaging object were marked. Distance between the planes and the $3 \mathrm{D}$ phantom was $1 \mathrm{~cm}$, and spacing between the measurement points was also $1 \mathrm{~cm}$. 


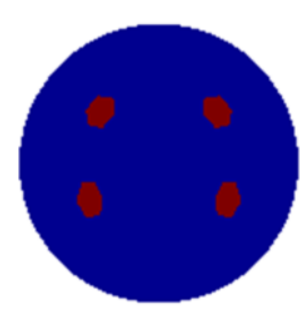

(a)

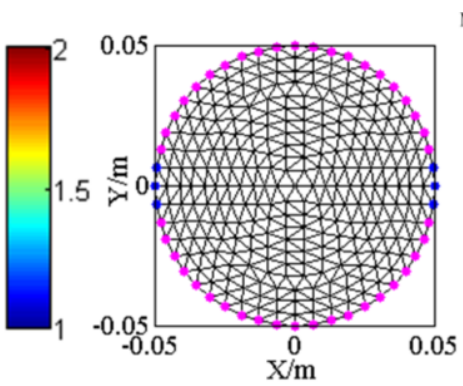

(b)

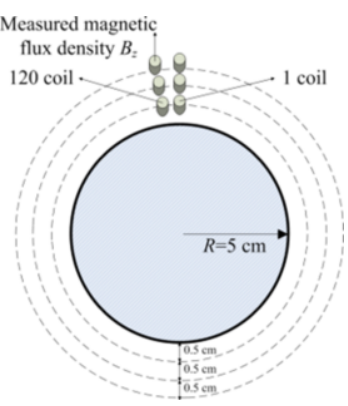

(c)

Fig. 1. The 2D simulated phantom. (a) Conductivity map of the model, (b) the opposite electrodes covering 2 elements located in the horizontal direction at the center, (c) the diagram displaying the positions of the coils.

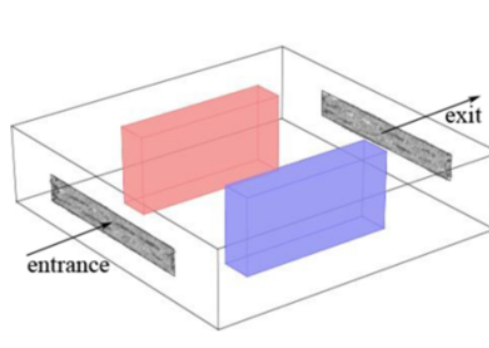

(a)

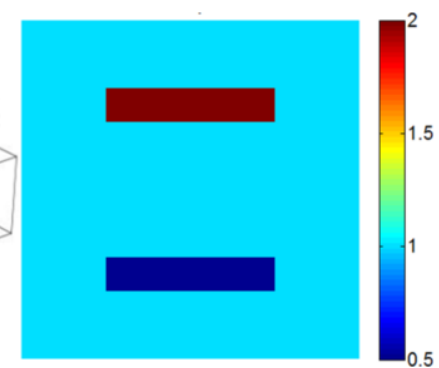

(b)

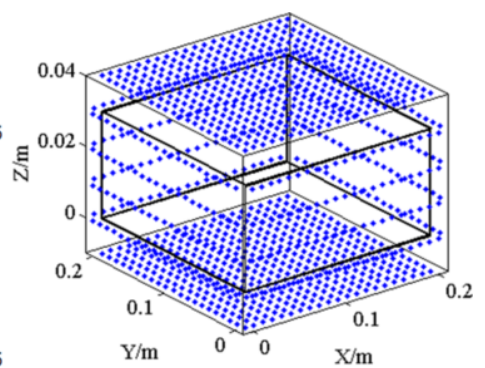

(c)

Fig. 2. The 3D simulated model. (a) The model with the electrodes, (b) tomographic conductivity map of the model, (c) the diagram displaying the positions of measurement points surrounding the object.

The numerical experiment for highlighting the convergence behaviour of the total variation regularized algorithm was performed. In the paper, the conductivity image was reconstructed from the simulated data with no noise, selecting $10^{-7}$ as the regularization parameter for the noise free case. The convergence behaviour of the TV regularized algorithm for the 2D phantom is illustrated in Figure 3 . The total variation regularized algorithm shows steady decrease in the error norm over the first 5 iterations. Convergence is achieved by the $5^{\text {th }}$ iteration - there was no appreciable change in the error norm and total variation after the $5^{\text {th }}$ iteration.

In actual measurements, there will be some necessary noises. In order to test the noise robustness of the reconstruction algorithms, white Gaussian noises with different noise levels were added to the simulated magnetic flux density data. The noise level was represented by signal-to-noise ratio (SNR) measured in $\mathrm{dB}$. Figure 4 illustrates the noise performance of $L^{2}$ norm and TV regularized algorithms for the 2D simulated phantom. For the quantitative evaluation of errors, the relative $L^{2}$ error of the reconstructed image is defined as follows,

$$
\operatorname{Re}=\left\|\sigma_{r}-\sigma_{t}\right\|_{2} /\left\|\sigma_{t}\right\|_{2}
$$

where $\sigma_{\mathrm{r}}$ is the reconstructed solution, $\sigma_{\mathrm{t}}$ is the true image. The relative errors of the above reconstructed images are shown in Table 1. For the 3D simulated phantom, Figure 5 displays the reconstructed conductivity images of the central slice using $L^{2}$ norm and TV regularized algorithms. 


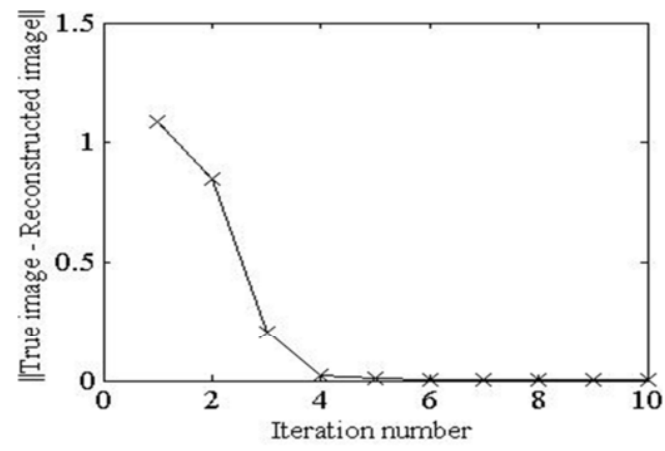

(a)

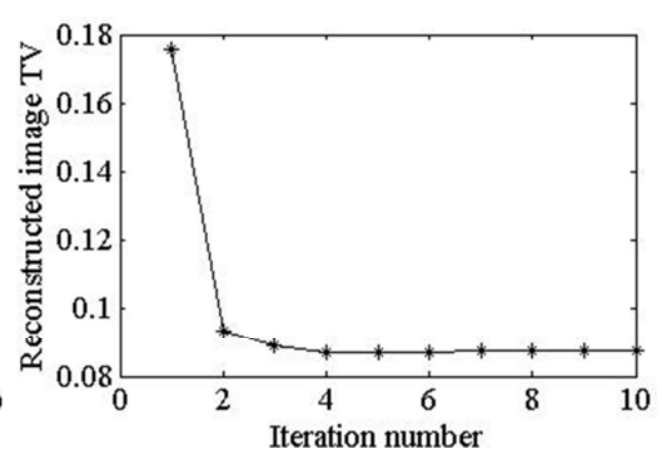

(b)

Fig. 3. The convergence behaviour of the TV regularization for the 2D phantom. (a) Difference between the true image and reconstructed image against the iteration number, (b) total variation against the iteration number.

Table 1

The relative error of the reconstructed conductivity images for different SNR

\begin{tabular}{llllll}
\hline SNR & $80 \mathrm{~dB}$ & $70 \mathrm{~dB}$ & $60 \mathrm{~dB}$ & $50 \mathrm{~dB}$ & $40 \mathrm{~dB}$ \\
\hline$L^{2}$ norm regularization & 0.1158 & 0.1265 & 0.1447 & 0.1671 & 0.1714 \\
TV regularization & 0.0078 & 0.0357 & 0.1176 & 0.1561 & 0.1649 \\
\hline
\end{tabular}

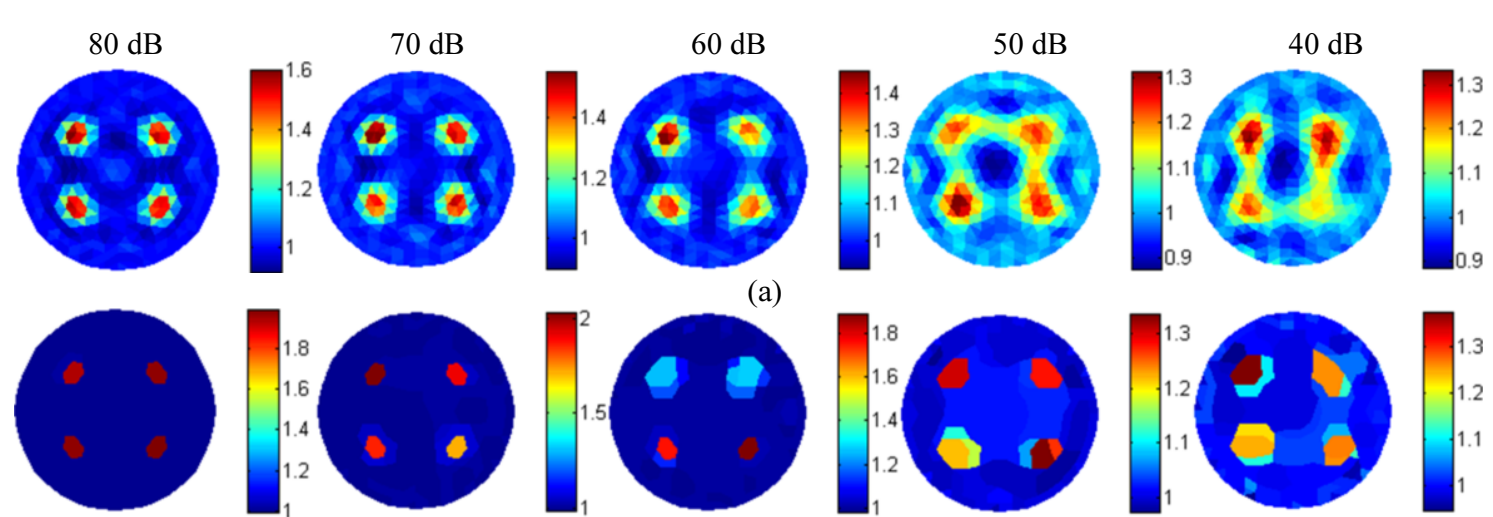

(b)

Fig. 4. Reconstructed conductivity images for $2 \mathrm{D}$ phantom. (a) The reconstructed results for $L^{2}$ norm regularized algorithm, (b) the reconstructed results for the TV regularized algorithm.

Even though noise was added to the simulated magnetic flux density, the conductivity can still be accurately reconstructed. It can be reconstructed with a satisfactory result when the SNR is superior to $60 \mathrm{~dB}$, while the results get worse when SNR is lower. Results show an exciting performance of robustness to the noise. The TV regularized algorithm produces the sharper images. Figure 6 shows the profile maps of the conductivity images produced by the $L^{2}$ norm regularized algorithm and the TV regularized algorithm along the horizontal lines through the two inclusions of the images. The TV regularized algorithm is capable of showing a better separation between the different inclusions, while the $L^{2}$ norm regularized algorithm shows a smoother transition, offering a less clear separation between inclusions. 
$70 \mathrm{~dB}$
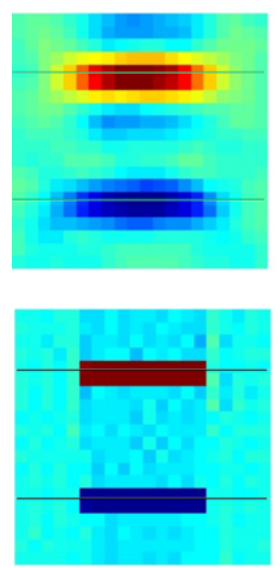

$60 \mathrm{~dB}$

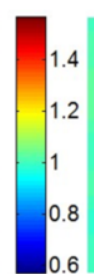

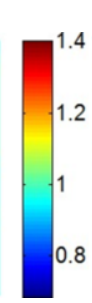

(a)

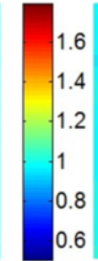

$50 \mathrm{~dB}$
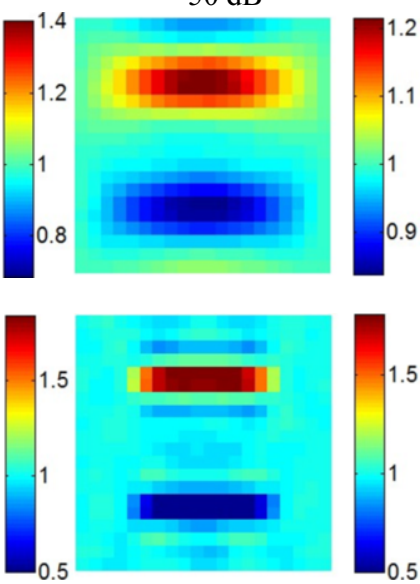

(b)

Fig. 5. Reconstructed conductivity images from the magnetic field data with different noise levels for 3D phantom. (a) The reconstructed results for $L^{2}$ norm regularized algorithm, (b) the reconstructed results for the TV regularized algorithm.

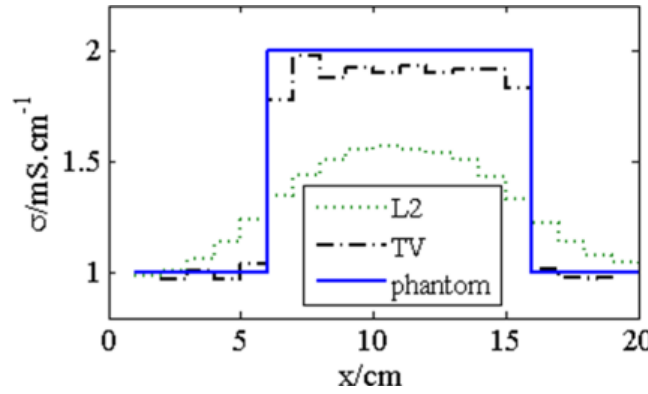

(a)

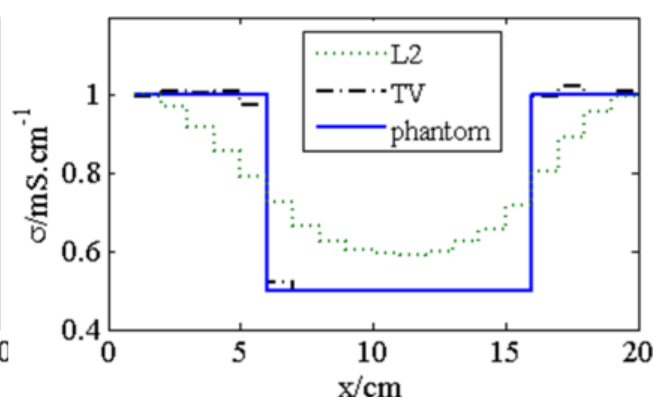

(b)

Fig. 6. Profile plots of the conductivity images along the horizontal lines through the two inclusions of the images for $70 \mathrm{~dB}$ shown in Figure 5. (a) The profile plots along the top lines, (b) the profile plots along the bottom lines.

\section{Conclusion}

In this work, the TV regularization is applied to the inverse problem of MDEIT. The primal dualinterior point method was employed for efficiently minimizing the TV penalty. Through the simulations of $2 \mathrm{D}$ and $3 \mathrm{D}$ conductivity image reconstructions, it is shown that the TV regularized algorithm produces sharper images compared with the traditional $L^{2}$ norm regularization, and has better robustness to noise.

The TV regularization leads to improvements in localizing the inhomogeneities in MDEIT. Furthermore, the sharp edges and piecewise constancy of the TV regularization may encourage the enhancements in clinical applications. For example, MDEIT may be useful in the detection of cancer that is the localized high impedance tissue. Additionally, MDEIT might improve current imaging techniques in the dynamic continuous real-time monitoring of the imaging object such as the brain. But MDEIT still struggles to produce images with good resolution that are routine in CT and MRI because of the inherent ill-posedness of MDEIT inverse problem. A larger number of injections and an improved set of coil positioning may lead to further improvements. 


\section{Acknowledgement}

This work was supported by the National Natural Science Foundation of China (No. 61374015, 61202258), the Ph.D. Programs Foundation of Ministry of Education of China (No. 20110042120037), the Fundamental Research Funds for the Central Universities (No. N110219001) and the Liaoning Provincial Natural Science Foundation of China (No. 201102067).

\section{References}

[1] T.J.C. Faes, H.A. Meij, J.C. Munck and R.M. Heethaar, The electric resistivity of human tissues (100 Hz-10 MHz): A meta-analysis of review studies, Physiological Measurement 20 (1999), R1-R10.

[2] R.H. Ireland and D.C. Barber, Constrained image reconstruction for magnetic detection electrical impedance tomography, International Journal of Imaging Systems and Technology 17 (2007), 379-382.

[3] R.H. Ireland, J.C. Tozer, A.T. Barker and D.C. Barber, Towards magnetic detection electrical impedance tomography: Data acquisition and image reconstruction of current density in phantoms and in vivo, Physiological Measurement 25 (2004), 775-796.

[4] D. Strong and T. Chan, Edge-preserving and scale-dependent properties of total variation regularization, Inverse Problem 19 (2003), S165-S187.

[5] G. Li, L. Hao, R. Chen and L. Lin, A new electrode mode for magnetic detection electrical impedance tomography, IEEE Transactions on Magnetics 48 (2012), 2543-2550.

[6] L. Hao, G. Li and L. Lin, Optimization of measurement arrangements for magnetic detection electrical impedance tomography, IEEE Transactions on Biomedical Engineering 61 (2014), 444-452.

[7] P.C. Hansen, Regularization tools: A Matlab package for analysis and solution of discrete ill-posed problems version 4.1 for matlab 7.3, Numerical Algorithms 46 (2007), 189-194.

[8] L.I. Rudin, S. Osher and E. Fatemi, Nonlinear total variation based noise removal algorithms, Physica D: Nonlinear Phenomena 60 (1992), 259-268.

[9] D. C. Dobson and F. Santosa, Recovery of blocky images from noisy and blurred data, SIAM Journal on Applied Mathematics 56 (1996), 1181-1198.

[10] E. Somersalo, J. Kaipio, M. Vauhkonen and D. Baroudi, Impedance imaging and markov chain monte carlo methods, Proceedings of the International Society for Optical Engineering 3171 (1997), 175-185.

[11] A. Borsic, B.M. Graham, A. Alder and W.R.B. Lionheart, In vivo impedance imaging with total variation regularization, IEEE Transactions on Medical Imaging 29 (2010), 44-54. 